\title{
Fetal hypoxemia causes abnormal myocardial development in a preterm ex utero fetal ovine model
}

\author{
Kendall M. Lawrence, ${ }^{1}$ Samson Hennessy-Strahs, ${ }^{2}$ Patrick E. McGovern, ${ }^{1}$ Ali Y. Mejaddam, ${ }^{1}$ \\ Avery C. Rossidis, ${ }^{1}$ Heron D. Baumgarten, ${ }^{1}$ Esha Bansal, ${ }^{2}$ Maryann Villeda, ${ }^{2}$ Jiancheng Han, ${ }^{1}$ \\ Zhongshan Gou, ${ }^{1}$ Sheng Zhao, ${ }^{1}$ Jack Rychik, ${ }^{3}$ William H. Peranteau, ${ }^{1}$ Marcus G. Davey, ${ }^{1}$ \\ Alan W. Flake, ${ }^{1}$ J. William Gaynor, ${ }^{4}$ and Carlo R. Bartoli ${ }^{2}$ \\ ${ }^{1}$ Center for Fetal Research, Department of Surgery, Children's Hospital of Philadelphia Research Institute, Philadelphia, \\ Pennsylvania, USA. 'Division of Cardiovascular Surgery, Department of Surgery, Hospital of the University of \\ Pennsylvania, Philadelphia, Pennsylvania, USA. ${ }^{3}$ Division of Cardiology, Department of Pediatrics, Children's \\ Hospital of Philadelphia, Philadelphia, Pennsylvania, USA. ${ }^{4}$ Division of Pediatric Cardiothoracic Surgery, Department \\ of Surgery, Children's Hospital of Philadelphia, Philadelphia, Pennsylvania, USA.
}

In utero hypoxia is a major cause of neonatal morbidity and mortality and predisposes to adult cardiovascular disease. No therapies exist to correct fetal hypoxia. In a new ex utero fetal support system, we tested the hypothesis that hypoxemic support of the fetus impairs myocardial development, whereas normoxic support allows normal myocardial development. Preterm fetal lambs were connected via umbilical vessels to a low-resistance oxygenator and placed in a sterilefluid environment. Control normoxic fetuses received normal fetal oxygenation, and hypoxemic fetuses received subphysiologic oxygenation. Fetuses with normal in utero development served as normal controls. Hypoxemic fetuses exhibited decreased maximum cardiac output in both ventricles, diastolic function, myocyte and myocyte nuclear size, and increased myocardial capillary density versus control normoxic fetuses. There were no differences between control normoxic fetuses in the fetal support system and normal in utero controls. Chronic fetal hypoxemia resulted in significant abnormalities in myocyte architecture and myocardial capillary density as well as systolic and diastolic cardiac function, whereas control fetuses showed no differences. This ex utero fetal support system has potential to become a significant research tool and novel therapy to correct fetal hypoxia.

Conflict of interest: The authors have declared that no conflict of interest exists.

License: Copyright 2018, American Society for Clinical Investigation.

Submitted: August 27, 2018 Accepted: October 29, 2018 Published: December 6, 2018

\section{Reference information:} JCI Insight. 2018;3(23): e124338. https://doi.org/10.1172/jici. insight.124338.

\section{Introduction}

In utero fetal hypoxia is a leading cause of neonatal morbidity and mortality (1). Common disorders of pregnancy such as intrauterine growth restriction, preeclampsia, gestational diabetes, and substance abuse may impair utero-placental function and lead to fetal hypoxia (2). Fetal hypoxia results in a 7 -fold increase in intrauterine death and 10-fold increase in 30-day mortality versus normoxic fetuses with physiologic oxygen levels (3). Fetuses that survive hypoxemic conditions are at high risk for perinatal and postnatal morbidity due to abnormal organ development and function such as occur in cardiovascular organs.

Indeed, a growing body of observational evidence has demonstrated a relationship between fetal hypoxia and an increased risk of heart failure, coronary artery disease, hypertension, and stroke in adulthood (4-9). These findings suggest that fetal hypoxia causes permanent, maladaptive cardiovascular abnormalities in utero that may persist into adult life and predispose patients to adult cardiovascular disease. However, the hypothesis that fetal hypoxia results in abnormal myocardial development and function remains untested in a controlled experimental system. Little mechanistic data exist to understand these complex relationships

Currently, no therapies exist to reverse most causes of fetal hypoxia. However, recent advances in low-resistance oxygenators allowed the development of a pumpless circulatory system to support fetuses outside of the maternal uterus (Figure 1) (10). With this system, preterm fetuses are removed from the mother via cesarean section and connected via umbilical vessels to the oxygenator. The fetal heart pumps blood out of the umbilical arteries through the extracorporeal circuit and low-resistance oxygenator and back to the fetus via the umbilical vein. As such, hemodynamics are modulated by the fetal cardiovascular system and not 
A

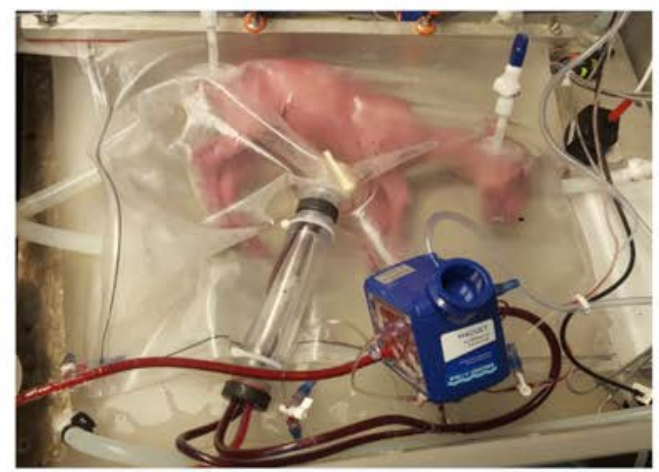

B

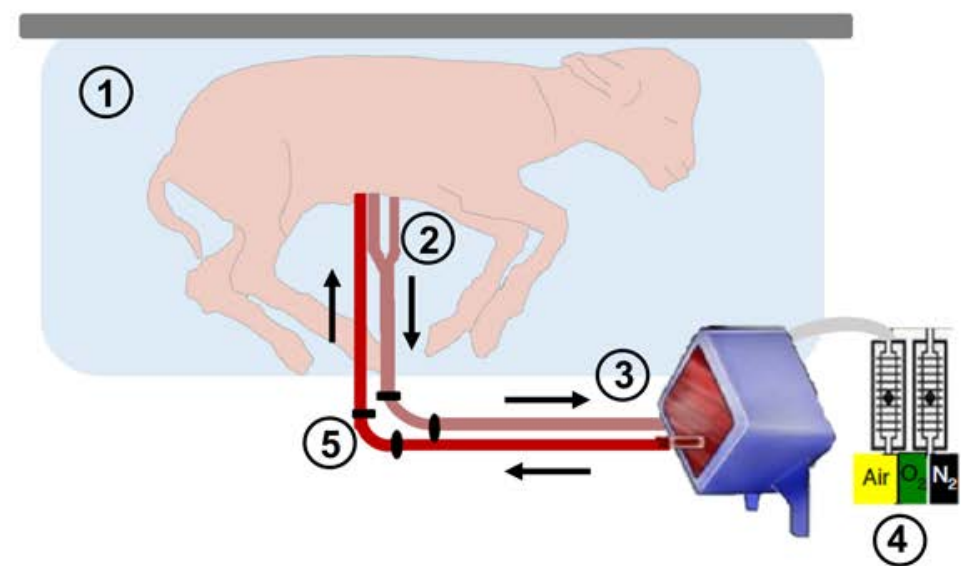

Figure 1. Ex utero fetal support system. A pumpless fetal circulatory support system was recently developed with an in-line, low-resistance oxygenator to support preterm, fetal lambs outside of the maternal uterus with normoxic conditions. In this system, over 4 weeks of support, fetuses have demonstrated normal somatic growth, organ development, and survival after removal from support. (A) A fetal lamb cannulated at 107 days of gestation is shown on day 1 of support. (B) Circuit components of the ex utero support system are shown. The fetal lamb rests in a sterile environment that provides continuous fluid exchange (1). The lamb is cannulated via two umbilical arteries and one umbilical vein (2), which are connected to a pumpless circuit and low-resistance oxygenator (3). The fetal circulation pumps blood to and from the oxygenator. Gas exchange is accomplished with blended sweep gas (4). Precise oxygen delivery is possible. Umbilical artery and vein pressure, flow, and oxygen saturations are measured via pressure transducers, a flow meter, and a spectrometer external to the circuit (5).

the support system. Prior studies with preterm, fetal lambs have shown that this ex utero fetal support system extended fetal gestation, allowed normal somatic growth and organ maturation outside of the maternal uterus, and led to survival after removal from support (10). Importantly, precise control of oxygen levels is possible. As such, this system has provided a unique opportunity to study fetal myocardial development ex utero during chronic hypoxemia versus control fetuses supported ex utero with normal oxygenation and control fetuses that developed normally in utero. The system may also have significant utility as a research tool to investigate multiple fetal pathologies in a highly controlled ex utero environment.

In this system, we tested the hypotheses that hypoxemic support of the fetus impairs myocardial development and cardiac function, whereas normoxic support of the fetus allows normal myocardial development and cardiac function. The goal of this study was 2-fold: (a) to explore relationships between fetal hypoxemia and myocardial development and function toward understanding mechanistic relationships between fetal hypoxemia and cardiovascular disease, and (b) to investigate whether preterm fetuses supported with normal oxygen levels develop normal myocardial architecture and function, which would have implications for the utility of this system as a therapy for intrauterine hypoxia.

\section{Results}

Fetal age. Gestational age at necropsy was similar between the hypoxemic $(n=7)$, control normoxic $(n=9)$, and control in utero $(n=8)$ fetuses $(128 \pm 2$ vs. $132 \pm 2$ vs. $135 \pm 2$ days, $P=0.19)$.

Oxygenation and acid/base status. Hypoxemic fetuses had significantly reduced venous ( $28 \pm 7$ vs. $55 \pm 9$ $\mathrm{mmHg}, P=0.04)$ and arterial $(15 \pm 2 \mathrm{vs.} 23 \pm 1 \mathrm{mmHg}, P<0.001)$ partial pressure of oxygen versus control normoxic fetuses. As a result, oxygen delivery was significantly reduced in hypoxemic fetuses versus control normoxic fetuses for the duration of the study (Figure $2 \mathrm{~A} ; 15 \pm 1 \mathrm{vs.} 23 \pm 1 \mathrm{ml} / \mathrm{kg} / \mathrm{min}, P<0.0001$ ).

Hypoxemic fetuses developed a persistent lactic acidosis ( $4.0 \pm 0.4$ vs. $1.4 \pm 0.3 \mathrm{mmol} / 1, P<0.0001)$ and base deficit $(-4.0 \pm 0.6$ vs. $-1.2 \pm 0.5 \mathrm{mEq} / 1, P<0.01)$, whereas control normoxic fetuses maintained normal acid/base status throughout the study (Figure 2B).

Systemic hemodynamic response to hypoxemia. Maximum cardiac output and the slope of the relationship between oxygen delivery and cardiac output were examined by comparing the best-fit lines individually in the right or left ventricle of hypoxemic versus control normoxic fetuses. In the left ventricle (Figure $3 \mathrm{~A}$ ), hypoxemic fetuses demonstrated a reduced maximum cardiac output response (blue intercept significantly lower than red intercept, $P<0.0001$ ) but a similar slope of the relationship between oxygen delivery and left 
A

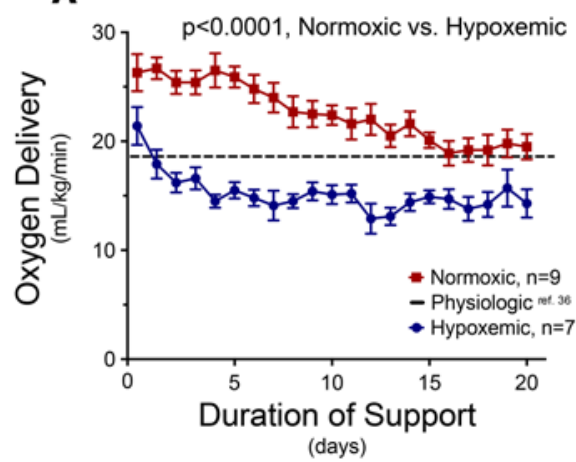

B

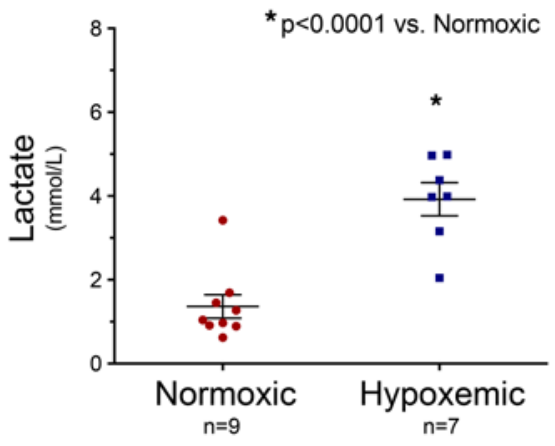

Figure 2. Establishment of normoxic versus hypoxemic conditions. Preterm fetal lambs were connected via umbilical vessels to a low-resistance oxygenator and placed in a sterile-fluid environment. (A) Control normoxic fetuses $(n=9)$ received physiologic oxygen levels for the duration of support ( $23 \pm 1 \mathrm{ml} / \mathrm{kg} / \mathrm{min}, 24 \pm 2$ days). In contrast, hypoxemic fetuses $(n=7)$ received subphysiologic levels of oxygen for the duration of support ( $15 \pm 1 \mathrm{ml} / \mathrm{kg} / \mathrm{min}, 18 \pm 2$ days). The dashed horizontal line demonstrates the threshold for normal in utero oxygen levels (36). Oxygen delivery was significantly lower in the hypoxemic group than in the normoxic group of fetuses $(P<0.0001)$. (B) As a result, mean serum lactate levels were significantly increased in hypoxemic fetuses compared with control normoxic fetuses for the duration of the study $(P<0.0001)$. Statistical comparisons were made across groups with unpaired, 2-tailed Student's $t$ tests.

ventricular cardiac output (similar slope of blue and red, $P=0.57$ ). In contrast, in the right ventricle (Figure 3B), both maximum cardiac output response (blue intercept significantly lower than red intercept, $P<$ 0.0001 ) and the slope of the relationship between oxygen delivery and right ventricular cardiac output (blue slope significantly lower than red slope, $P=0.02$ ) were reduced.

Fetal body and heart weights. Body weight tended to be lower in hypoxemic fetuses compared with control normoxic fetuses and control in utero fetuses $(2.4 \pm 0.3$ vs. $3.1 \pm 0.3 \mathrm{~kg}$ and $3.4 \pm 0.3, P=0.12)$. Heart weight was significantly reduced in hypoxemic fetuses compared with control normoxic fetuses and control in utero fetuses $(19 \pm 2$ vs. $28 \pm 3$ and $30 \pm 3 \mathrm{~g}, P=0.03)$. There was no significant difference in heart weight between control normoxic fetuses and control in utero fetuses.

Myocardial histology. Hypoxemic fetuses demonstrated significantly smaller myocytes than control normoxic fetuses and in utero control fetuses (Figure 4, A-D; $32 \pm 2$ vs. $49 \pm 5$ and $46 \pm 3 \mu \mathrm{m}^{2}, P<0.01$ ). Similarly, hypoxemic fetuses exhibited significantly smaller nuclei than control normoxic fetuses (Figure 4, E-H; $16 \pm 1$ vs. $\left.19 \pm 1 \mu \mathrm{m}^{2}, P=0.01\right)$. In contrast, myocyte size and myocyte nuclear size were not different between control normoxic and control in utero fetuses.

Hypoxemic fetuses demonstrated a significant increase in capillary density compared with in utero control fetuses (Figure 5, A-D; $258 \pm 26$ vs. $176 \pm 20$ capillaries per high-power field $[\mathrm{HPF}], P=0.02$ ) and control normoxic fetuses ( $258 \pm 26$ vs. $157 \pm 17$ capillaries/HPF, $P<0.01)$. In contrast, myocardial capillary density was not different between control normoxic fetuses and control in utero fetuses. There was no statistically significant difference in myocardial apoptosis across groups (Figure 5, E-H).

Diastolic function as measured by the mitral E/A ratio $(0.73 \pm 0.02$ vs. $0.64 \pm 0.02, P<0.01)$ and tricuspid E/A ratio $(0.71 \pm 0.01$ vs. $0.65 \pm 0.01, P<0.01)$ were significantly decreased in hypoxemic fetuses versus normoxic control fetuses.

\section{Discussion}

Previously, challenges of studying fetal development in utero have prevented continuous, long-term study of myocardial development during chronic hypoxemic conditions. Prior animal models of intrauterine hypoxemia (partial carunclectomy, single umbilical artery ligation, maternal hypoxemia) have not been able to achieve subphysiologic oxygen delivery without concurrent reductions in nutritional delivery, alterations in cardiac afterload, or maternal stress (11-13). In contrast, we were able to produce hypoxemic conditions while minimizing confounding variables to determine the impact of hypoxemia alone on myocardial development. Indeed, our pumpless fetal circulatory support system was recently developed with an in-line, low-resistance oxygenator to support preterm, fetal lambs outside of the maternal uterus with 

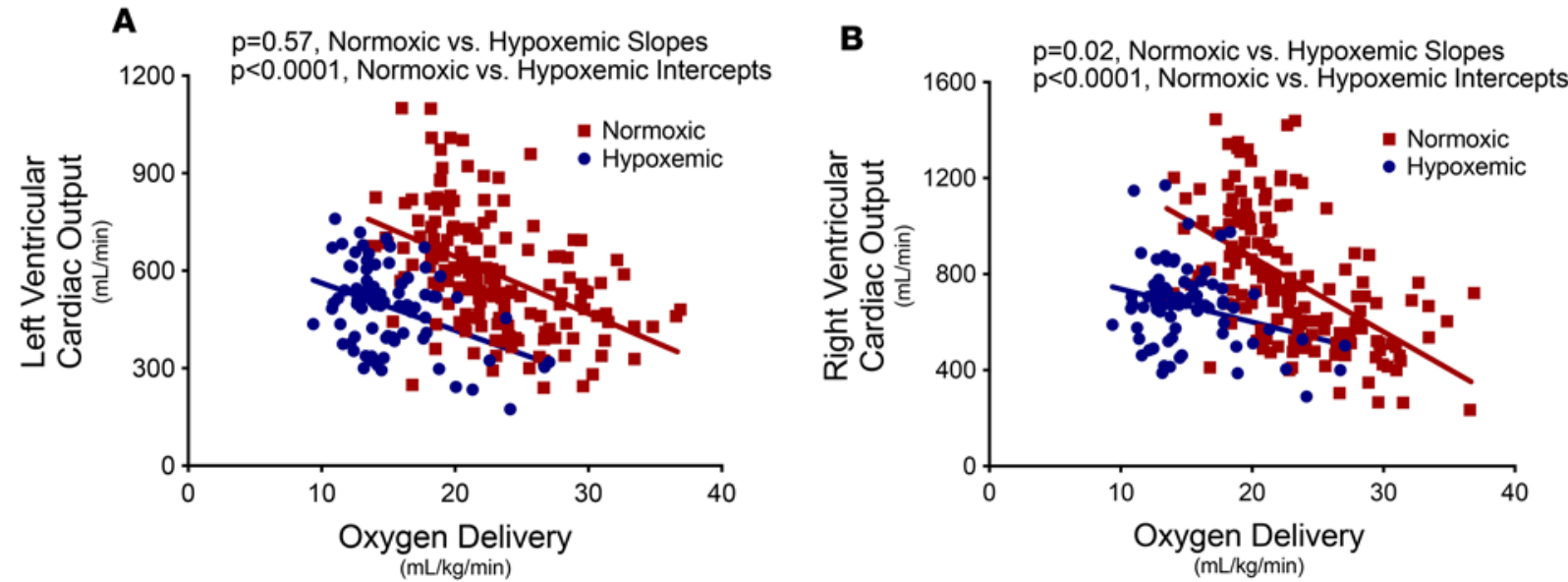

Figure 3. Left and right ventricular cardiac dynamics in response to oxygen delivery. Preterm fetal lambs that received either normal oxygen delivery ( $n=9,23 \pm 1 \mathrm{ml} / \mathrm{kg} / \mathrm{min}, 24 \pm 2$ days) or subphysiologic oxygen delivery $(n=7,15 \pm 1 \mathrm{ml} / \mathrm{kg} / \mathrm{min}, 18 \pm 2$ days) in an ex utero fetal support system demonstrated differential cardiac output responses to oxygen delivery. The relationship between ventricular cardiac output and oxygen delivery are shown for each ventricle. (A) In the left ventricle, hypoxemic fetuses demonstrated a reduced maximum cardiac output response $(P<0.0001)$ but a similar slope of the relationship between oxygen delivery and left ventricular cardiac output $(P=0.57)$ versus normoxic controls. (B) In contrast, in the right ventricle, both maximum cardiac output response $(P<0.0001)$ and the slope of the relationship between oxygen delivery and right ventricular cardiac output $(P=$ 0.02 ) were reduced versus normoxic controls. Linear regression was performed to compare intercepts and slopes of the best-fit lines for the relationship between fetal oxygen delivery and cardiac output.

normoxic conditions (10). In this system, lambs have been successfully supported for 4 weeks and demonstrated normal somatic growth, organ development, and survival after removal from support. The system allows for precise control of oxygen delivery to the supported fetus. As such, this system permitted us to explore effects of chronic fetal hypoxemia on myocardial development and function compared with control fetuses supported with normal oxygenation and fetuses that developed normally in utero. The system has utility as a powerful research tool to investigate many other fetal pathologies.

In this system, we demonstrated that (a) chronic hypoxemic support of the preterm fetus resulted in significant abnormalities in myocardial architecture and myocardial capillary density as well as systolic and diastolic cardiac function, whereas (b) normoxic support of the preterm fetus resulted in normal myocardial structure and function. These findings may inform mechanisms of postnatal cardiac pathology related to intrauterine hypoxia with important implications for infant and adult life. Additional studies will define a potential role for ex utero fetal mechanical circulatory support as a rescue therapy for premature birth and in utero hypoxia.

Cardiac effects of chronic fetal hypoxemia. An ex utero support system was recently developed to extend fetal gestation, allow normal, somatic growth and organ maturation outside of the maternal uterus, and allow immediate access to the fetus for continuous assessment (10). In this system, we controlled fetal oxygen delivery. In fetal sheep exposed to 3 weeks of chronic hypoxemia, metabolic demand exceeded oxygen supply. Fetuses transitioned to a state of partial anaerobic metabolism. Lactate levels increased, and a base deficit developed. For any given lower oxygen delivery rate (lower $\left.\mathrm{PaO}_{2}\right)$, left and right ventricular cardiac output were lower in hypoxemic fetuses and suggested reduced capacity to meet metabolic demands than control normoxic fetuses. A reduced slope of the linear response of cardiac output to oxygen delivery was observed in the right ventricle, which supplies blood flow to the body and vital organs in the fetal circulation, but not the left ventricle, which supplies blood flow to the fetal heart and brain. Preferential left ventricular compensation, which has previously been reported in chronically hypoxemic fetal sheep (14-16) and humans (17), was likely a response to protect blood flow to the developing fetal heart and brain. Indeed, there is evidence that chronic in utero hypoxemia leads to regional redistribution of systemic flow to maintain normal blood flow to the heart and brain in late gestation (18).

Hypoxemic fetuses demonstrated significant differences in heart size and myocyte architecture compared with both control fetus groups. Heart weights of hypoxemic fetuses were low. Histologically, myocyte and myocyte nuclear size were significantly decreased. Reduced myocyte growth suggested downregulation of anabolic pathways in the hypoxemic myocardium, a phenomenon well known to occur when aerobic metabolism is limited (19). We also observed an increase in myocardial capillary density, which is a well- 
A

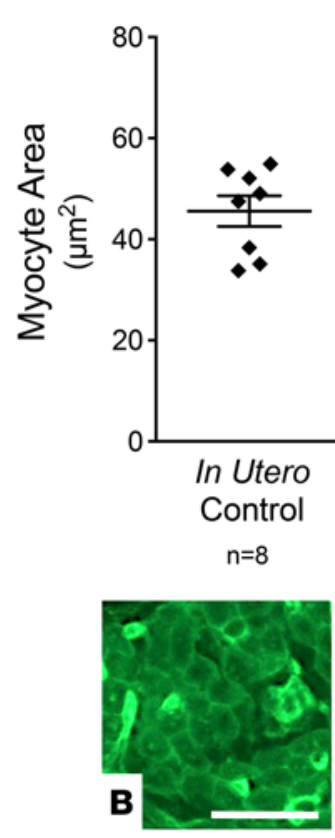

${ }^{*} p<0.01$ vs. In Utero Control $\dagger_{p}<0.01$ vs. Normoxic

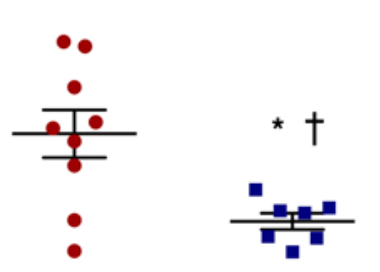

Normoxic Hypoxemic

$n=9$

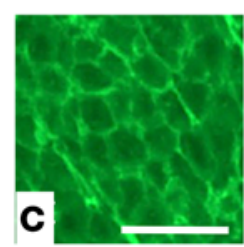

$\mathrm{n}=7$

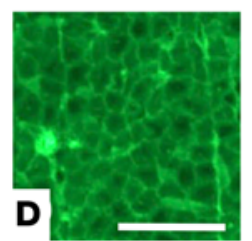

$\mathbf{E}$

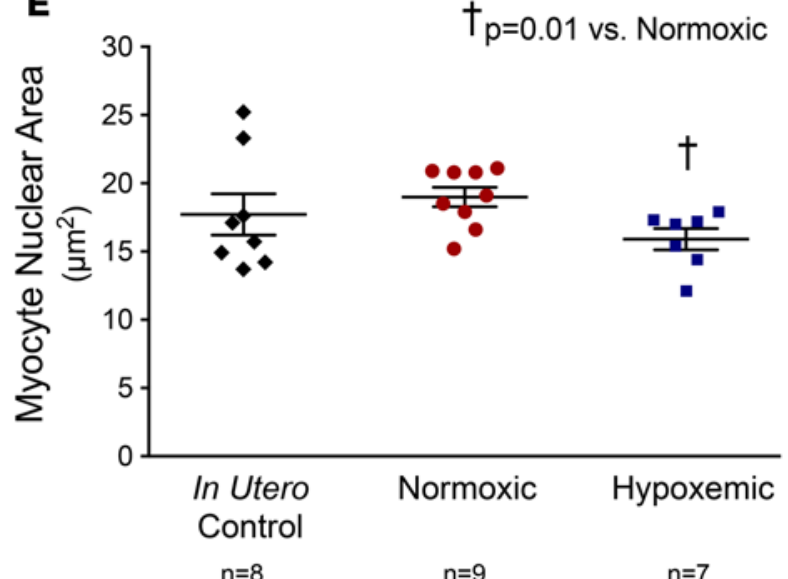

$n=8$
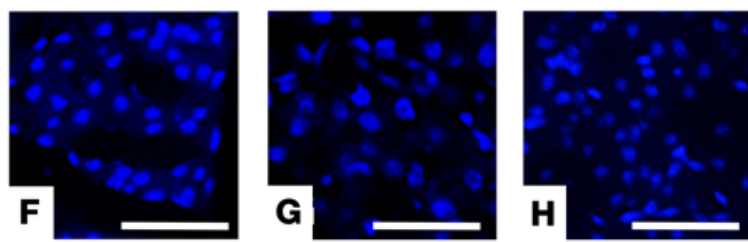

Figure 4. Myocyte characteristics. Preterm fetal lambs received either normal oxygen delivery ( $n=9,23 \pm 1 \mathrm{ml} / \mathrm{kg} / \mathrm{min}, 24 \pm 2$ days) or subphysiologic oxygen delivery ( $n=7,15 \pm 1 \mathrm{ml} / \mathrm{kg} / \mathrm{min}, 18 \pm 2$ days) in an ex utero fetal support system. At necropsy, myocardial histology was performed with hypoxemic and normoxic control hearts. Results were compared to a group of control hearts from fetuses raised normally in utero $(n=8)$. (A) Left ventricular myocytes from control in utero fetuses (B) and control normoxic fetuses (C) were significantly larger than myocytes from hypoxemic fetuses (D). There was no difference in myocyte size between control in utero fetuses and control normoxic fetuses. Original magnification, $\times 40$. Scale bars: $20 \mu \mathrm{m}$. (E) Left ventricular myocyte nuclei from control in utero fetuses $(\mathbf{F})$ and control normoxic fetuses (G) were larger than hypoxemic fetuses (H). There was no difference in myocyte nuclear size between control in utero fetuses and control normoxic fetuses. Original magnification, $\times 20$. Scale bars: $40 \mu \mathrm{m}$. Statistical comparisons were made across groups with 1-way analysis of variance with Tukey's post hoc tests to compare means between individual groups.

known compensatory response to tissue hypoxia. Previously in this system, during hypoxemic conditions, elevated levels of plasma hypoxia inducible factor-1a, a potent promoter of angiogenesis, were observed (20) and may have contributed to increased myocardial neovascularization. In hypoxemic fetuses, reduced myocyte growth and increased myocardial angiogenesis favorably rebalanced myocardial metabolic supply and demand. However, reduced myocyte size and myocardial hypervascularization in utero have both been shown to permanently alter cardiac structure (21-23). The development of abnormal myocardial architecture likely explains the relative diastolic dysfunction that we observed in the hypoxemic fetuses and has also previously been demonstrated in hypoxic human fetuses $(24,25)$. These findings support a mechanistic relationship between abnormal myocardial architecture in the fetal period that may persist into adulthood and elevated postnatal cardiovascular risk (4, 7, 26-28).

Importantly, increased myocardial apoptosis was not observed. In the heart, myocyte apoptosis is rare unless there is severe myocardial damage or disease. As such, cells in the myocardium observed to be undergoing apoptosis are frequently transient inflammatory cells that oversee the maintenance of myocardial architecture (29). The absence of apoptotic cells in either the hypoxemic or control normoxic groups suggests that severe myocardial damage was not present and that the ex vivo fetal support system did not cause significant myocardial inflammation.

Normoxic support of the fetus allowed normal myocardial development. Normoxic support led to myocardial development that was grossly and histologically similar to in utero controls. Heart weight, myocyte and myocyte nuclear size, myocardial capillary density, and myocardial apoptosis were not different between control normoxic fetuses and age-matched control in utero fetuses. These data support previous data to suggest that cardiac function is similar between fetuses that receive ex utero normoxic support and control fetuses that develop in utero (10).

These findings highlight the potential of this system as a novel therapy to support extremely premature infants or as a rescue therapy for fetuses suffering from in utero hypoxia, for which there are currently no therapies. 


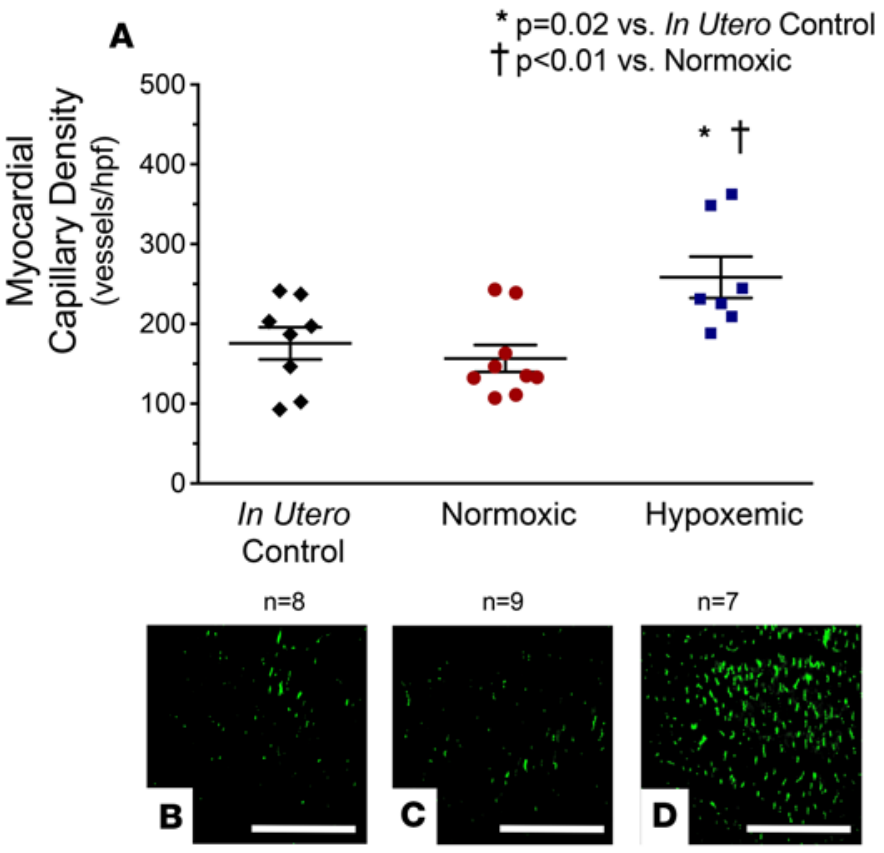

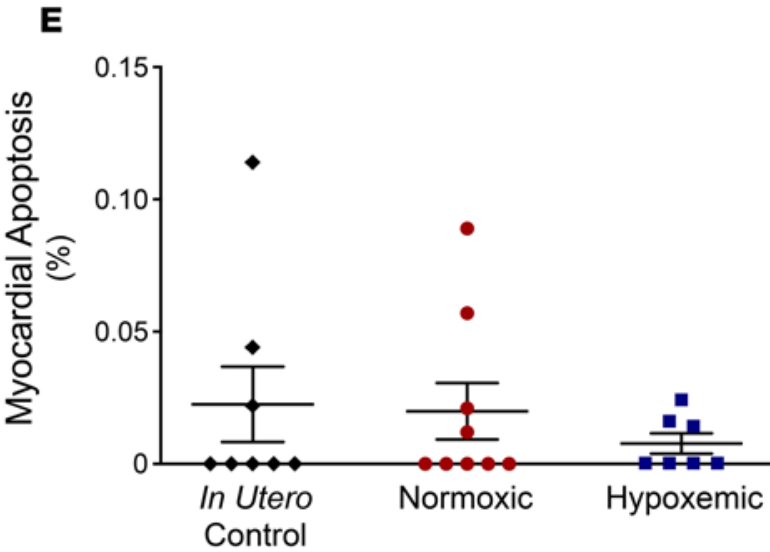

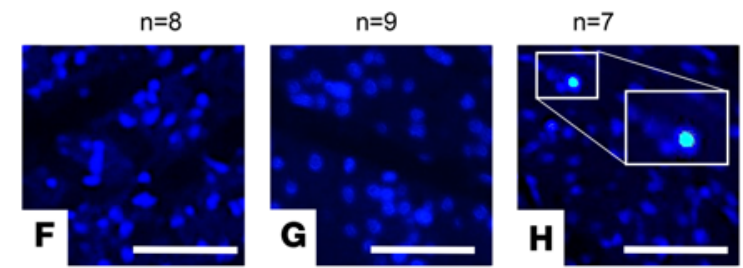

Figure 5. Myocardial histology. Preterm fetal lambs received either normal oxygen delivery $(n=9,23 \pm 1 \mathrm{ml} / \mathrm{kg} / \mathrm{min}, 24 \pm 2$ days) or subphysiologic oxygen delivery ( $n=7,15 \pm 1 \mathrm{ml} / \mathrm{kg} / \mathrm{min}, 18 \pm 2$ days) in an ex utero fetal support system. At necropsy, myocardial histology was performed and compared to a group of control fetuses raised normally in utero $(n=8)$. (A) Myocardial capillary density was significantly reduced in control in utero fetuses (B) and control normoxic fetuses (C) compared with hypoxemic fetuses (D). There was no difference in myocardial capillary density between the control in utero fetuses and control normoxic fetuses $(P=0.48)$. Original magnification, $\times 20$. Scale bars: $200 \mu \mathrm{m}$. (E-H) No difference in myocardial apoptosis was observed across experimental groups. A single TUNEL ${ }^{+}$nucleus is shown (F inset). Original magnification, $\times 40$. Scale bars: $40 \mu \mathrm{m}$. Statistical comparisons were made across groups with 1-way analysis of variance with Tukey's post hoc tests to compare means between individual groups.

Scalability and clinical observations. We do not anticipate that scalability will be a problem with this system. Human fetuses and fetal lambs have comparable size and physiology. The average birth weight of a lamb is 3.5 to $4.5 \mathrm{~kg}$, whereas the average birth weight of a human infant is 3 to $5 \mathrm{~kg}$. Likewise, humans and lambs have similar umbilical blood flow requirements of approximately 180 to $200 \mathrm{ml} / \mathrm{kg} / \mathrm{min}$. Lambs at 85 to 95 days gestational age are equivalent in weight to 23 - to 27 -week human fetuses ( $~ 500$ to $850 \mathrm{~g}$ ). Importantly, lambs at 85 to 95 days gestational age have been successfully cannulated and supported with this system (30), which demonstrates feasibility of support of human fetuses of a variety of sizes and weights.

We saw no evidence of poor hemocompatibility in this study or previous studies with this model (31, 32). During experiments, we did not observe evidence of hemolysis or thrombus formation. After each experiment, examination of the oxygenator and circuit tubing did not reveal evidence of gross thrombus. At necropsy, we did not observe evidence of thromboembolic events in the end organs. We attribute this to continuous systemic heparinization (target activated clotting time 180 to 200 seconds), the small surface area of circuit components, heparinized coating of circuit components, and the pumpless design of the system that avoids pump-related blood trauma.

Limitations. A potential limitation was the small number of fetuses studied. As such, there is a risk that observed effects were driven by chance findings from a few samples (type I statistical error). Encouragingly, fetuses demonstrated consistent and robust biological changes across multiple overlapping metabolic, hemodynamic, ultrasonographic, gross anatomic, and microscopic histologic parameters. Nonetheless, with a small sample size, we may have failed to detect important differences between groups (type II statistical error). The lack of borderline statistically significant $P$ values $(P \approx 0.10)$ suggested that this was not the case.

End-organ blood flow was not measured. In ongoing experiments, vascular responses and the regional redistribution of systemic flow are being examined to further explore how blood flow to the brain and heart are preserved in fetuses in late gestation and other pathologic conditions.

The resuscitation and management of individual fetuses varied. Administration of blood and fluids and correction of electrolytes were titrated on an individual basis in order to keep each fetus alive. Although 
a potential scientific limitation, in a clinical context this limitation may be viewed as a potential strength of the study. We expect that if this system were used for clinical therapy, patient management would be variable depending on the clinical situation. Therefore, our findings, which were robust across hypoxemic fetuses versus control normoxic fetuses despite variable management, may be interpreted in the clinical context in which variable patient management would be expected.

Conclusions. Recently, an ex utero support system was developed to support preterm fetuses outside of the maternal uterus. Previous studies with preterm, fetal lambs in this system demonstrated normal growth and organ development as well as survival after removal from support. Precise control of oxygen delivery to the fetus is possible, which allowed us to study effects of chronic hypoxemia on fetal myocardial development and function. Findings were compared to concurrent control fetuses supported with normal oxygen levels and control fetuses that developed normally in utero. Chronic fetal hypoxemia resulted in significant abnormalities in myocardial architecture and myocardial capillary density as well as systolic and diastolic cardiac function versus control fetuses. Abnormal myocardial architecture in the fetal period that persists into adulthood may be an important mechanistic link between fetal hypoxia and adult heart disease. Furthermore, this system has great potential as a powerful research tool to investigate many other fetal pathologies.

No current therapies exist to correct fetal hypoxia in utero. In our ex utero fetal support system, normoxic support allowed normal myocardial development. This system has the potential to become a significant novel therapy to correct fetal hypoxia. Additional studies will define a potential role for ex utero fetal mechanical circulatory support as a rescue therapy for in utero fetal hypoxia and further inform the nascent field of fetal mechanical circulatory support.

\section{Methods}

Animals and surgical procedure. Time-dated pregnant ewes were obtained from a local sheep vendor (May Family Enterprises) approved by the Children's Hospital of Philadelphia (CHOP) IACUC.

The surgical procedure was previously described in detail (10).

Preterm fetal lambs ( $n=16$, gestational age $109 \pm 1$ days; normal term ovine gestation is approximately 145 days) were removed from their mothers via cesarean section. For comparison, 105 to 115 days gestational age lambs are developmentally similar to a 23- to 27-week human fetus.

Umbilical arteries and vein were cannulated with 12-French cannulas and connected to a pumpless, low-resistance oxygenator (Quadrox-ID Neonatal Oxygenator, Maquet). Fetuses were then placed in a sterile, silver-impregnated bag containing a warm, sterile balanced salt physiologic solution $\left(109 \mathrm{mM} \mathrm{Na}^{+}, 104\right.$ $\mathrm{mM} \mathrm{Cl}^{-}, 19 \mathrm{mM} \mathrm{HCO}_{3}^{-}, 6.5 \mathrm{mM} \mathrm{K}^{+}$, and $1.6 \mathrm{mM} \mathrm{Ca}^{2+}$ ) that was continuously infused to maintain a fluid environment reminiscent of the maternal uterus (Figure 1).

Fetal care and monitoring. The care, metabolic, and continuous hemodynamic monitoring of preterm fetal lambs in the ex utero support system have previously been reported (10).

Fetuses were continuously supported with intravenous fluids and total parenteral nutrition that contained amino acids (10\% TrophAmine, titrated to blood urea nitrogen of $30 \mathrm{mg} / \mathrm{dl}$ ), lipids ( $20 \%$ Intralipid, 0.1 to 0.2 $\mathrm{g} / \mathrm{kg}$ /day), dextrose, and iron (titrated to plasma iron 200 to $300 \mu \mathrm{g} / \mathrm{dl}$ ). Insulin was administered as needed to maintain glucose at 30 to $40 \mathrm{mg} / \mathrm{dl}$. We ensured that normoxic and hypoxic fetuses had similar dextrose, protein, and lipid calories so that observed differences could not be attributed to nutritional differences. Furthermore, we ensured that calories delivered were similar to those delivered in utero to age-matched fetuses (33).

Fetuses received heparin (10 to 400 units/h) with a target activated clotting time of 180 to 200 seconds to prevent clot formation in the oxygenator and circuit. Fetal shunts were maintained open with prostaglandin E1 $(0.1 \mu \mathrm{g} / \mathrm{kg} / \mathrm{min})$. Recombinant erythropoietin (800 units $/ \mathrm{kg}$ ) was administered daily to promote fetal erythropoiesis. Fetuses were treated with blood transfusion and intravenous fluids as needed. Fetal blood pressure, heart rate, oxygen saturation, circuit blood flow, and sweep gas were continuously monitored and recorded (LabChart 7, AD Instruments Inc.).

Experimental groups. Three groups were studied (Table 1): (a) ex utero hypoxemic fetuses $(n=7),(b)$ ex utero normoxic control fetuses $(n=9)$, and (c) age-matched control fetuses raised in utero $(n=8)$. Data from some of the normoxic fetuses included in these experiments have previously been reported (10).

In hypoxemic fetuses, after a 24-hour stabilization period, oxygen content was regulated to maintain oxygen delivery at 14 to $16 \mathrm{ml} / \mathrm{kg} / \mathrm{min}$, which is consistent with reported levels of in utero hypoxemia (34, 35). In normoxic control fetuses, oxygen content was regulated to maintain physiologic oxygen delivery at greater than $19 \mathrm{ml} / \mathrm{kg} / \mathrm{min}$ (36). Oxygen delivery was continuously calculated and displayed and mon- 
Table 1. Ex utero support system

\begin{tabular}{|c|c|c|c|c|}
\hline & In Utero Control & Normoxic Control & Hypoxemic & $P$ value \\
\hline$n$ & 8 & 9 & 7 & - \\
\hline Gestational Age (days) & $135 \pm 2$ & $132 \pm 2$ & $128 \pm 2$ & 0.19 \\
\hline Arterial Oxygen (mmHg) & Physiologic & $23 \pm 1$ & $15 \pm 2$ & $<0.001$ \\
\hline Venous Oxygen (mmHg) & Physiologic & $55 \pm 9$ & $28 \pm 7$ & 0.04 \\
\hline Lactic Acid (mmol/l) & Physiologic & $1.4 \pm 0.3$ & $4.0 \pm 0.4$ & $<0.0001$ \\
\hline Base Deficit (mEq/l) & Physiologic & $-1.2 \pm 0.5$ & $-4.0 \pm 0.6$ & $<0.01$ \\
\hline Body Weight (kg) & $3.4 \pm 0.3$ & $3.1 \pm 0.3$ & $2.4 \pm 0.3$ & 0.12 \\
\hline Heart Weight (g) & $30 \pm 3$ & $28 \pm 3$ & $19 \pm 2$ & 0.03 \\
\hline
\end{tabular}

itored in LabChart according to the following equation: oxygen delivery $(\mathrm{ml} / \mathrm{min} / \mathrm{kg})=$ weight-adjusted umbilical blood flow $\times(1.34 \times \mathrm{Hb} \times$ postoxygenator oxygen saturation $)+\left(0.0031 \times\right.$ postoxygenator $\left.\mathrm{PaO}_{2}\right)$.

Umbilical blood flow was measured continuously via ultrasonic flow-probe (HXL Tubing Flowsensor, Transonic Systems Inc.). Noninvasive pre- and postmembrane oxygen saturations were measured continuously via oxygen sensor (M2-Sensor, Spectrum Medical). Twice-daily blood gases (Avoximeter 1000, Accriva Diagnostics) were obtained. Body weights were measured at the beginning and end of each study and growth rates were assumed to be exponential according to the formula $y=a e^{b x}$ (a, starting weight; $b$, growth rate in $\mathrm{g} / \mathrm{kg}$ per day). Daily weights were extrapolated from the exponential growth rate and used in the above equation to determine weight-adjusted flows and oxygen delivery.

When oxygen delivery deviated from target ranges, an auditory alarm triggered a trained technician to respond to increase or decrease the oxygen content of the sweep gas until oxygen delivery returned to within the target range. Sweep gas was a mixture of nitrogen, room air, and oxygen.

Fetuses supported in the ex utero system were euthanized at a gestational age of $131 \pm 2$ days. Agematched control fetal lambs ( $n=8$, gestational age $135 \pm 2$ days) were delivered via caesarian section, euthanized, and served as control fetuses that developed in utero for histologic comparisons. Euthanasia was performed with pentobarbital/phenytoin sodium $(117 \mathrm{mg} / \mathrm{kg})$.

Echocardiography. Echocardiographic examination was performed daily by a fetal echocardiographer with a Philips iE33 echocardiographic machine (Philips Healthcare). Standard windows were obtained with a S8-3 Transducer (8-3 MHz) across the sonolucent bag.

Calculated cardiac parameters included left ventricular (LV) and right ventricular (RV) cardiac output by measuring cross-sectional areas and Doppler-derived velocity-time integral tracings across aortic and pulmonic valves. LV and RV stroke volume were defined by ventricular output divided by heart rate. Peak flow velocities in early (E) and late (A) diastole across the atrioventricular valves were measured to calculate the E/A ratio, as an index of diastolic function (37).

Myocardial histology. At necropsy, fetal and cardiac weights were obtained. Hearts were fixed in $10 \%$ neutral buffered formalin. Fixed cross sections of LV myocardium were embedded in paraffin, sectioned at $5 \mu \mathrm{m}$, deparaffinized, rehydrated, and stained as below. Randomly selected photomicrographs were obtained from each sample with an EVOS FL Auto 2 microscope (Life Technologies). Images were analyzed with ImageJ software (NIH).

Mean myocyte size and myocyte nuclear size were quantified with FITC-conjugated wheat germ agglutinin and DAPI nuclear costaining (Life Technologies) to delineate the cell membrane and nucleus in at least 100 cross-sectional myocytes with centrally located nuclei.

Myocardial capillary density was quantified with FITC-conjugated isolectin-B4 staining (Sigma-A1drich). Three representative micrographs were obtained for each sample. Capillary density was defined as the number of capillaries per HPF. Values for each image were averaged to obtain a single representative mean value per tissue section $(38,39)$.

Myocardial apoptosis was determined with the DeadEnd Fluorometric TUNEL System (Promega), which catalytically incorporates fluorescein-12-dUTP at DNA strand breaks in cells actively undergoing programmed cell death. Nuclei were counterstained with DAPI. Overlaid FITC/DAPI foci were identified as TUNEL-positive nuclei $(29,38)$. The average rate of myocardial apoptosis was calculated from approximately 12,000 myocytes per myocardial sample. 
Statistics. Prism version 6.0 (GraphPad Software) was used to perform statistical analyses and plot data. Daily hemodynamic, echocardiographic, and laboratory measurements were averaged for each fetus. Groups were compared with unpaired, 2-tailed Student's $t$ tests. One-way analysis of variance with Tukey's post hoc test was performed when 3 groups were compared. Linear regression was performed to compare slopes of the best-fit lines for the relationship between fetal oxygen delivery and cardiac output. A $P$ value $<$ 0.05 was considered statistically significant. All data are presented as mean \pm standard error.

Study approval. This study was conducted in accordance with the NIH guidelines for the care and use of animals in research. All experimental procedures were approved by the IACUC at The Children's Hospital of Philadelphia Research Institute.

\section{Author contributions}

KML was involved in all aspects of the study and drafted the manuscript. SHS participated in data collection, analyzed data, and assisted in drafting the manuscript. PEM, EB, and MV participated in data collection, analysis, and interpretation. AYM, ACR, HDB, JH, ZG, and SZ contributed to animal data collection. JR participated in data interpretation and edited the manuscript. WHP participated in model development, animal surgeries, and edited the manuscript. MGD, AWF, and JWG were involved in experimental design and edited the manuscript. CRB was involved in histologic experimental design, data analysis and interpretation, and drafted the manuscript.

\section{Acknowledgments}

We acknowledge and thank the following individuals for contributions to this work and technical support: Robert Dowling, Jacqueline Amato, Grace Hwang, Aaron Weilerstein, and Kathleen Young. This work was supported by the CHOP Institutional Development Fund, the CHOP Departments of Surgery and Cardiothoracic Surgery, and the Mortimer J. Buckley Endowed Chair in Cardiothoracic Surgery.

Address correspondence to: Carlo R. Bartoli, Hospital of the University of Pennsylvania, Department of Surgery, Division of Cardiovascular Surgery, 380 South University Avenue, Hill Pavilion, Room 410B, Philadelphia, Pennsylvania 19104, USA. Email: carlo.bartoli@uphs.upenn.edu.

1. [No authors listed]. Neonatal and perinatal mortality: country, regional and global estimates. World Health Organization. http://www.who.int/iris/handle/10665/43444. Accessed November 9, 2018.

2. Giussani DA. The fetal brain sparing response to hypoxia: physiological mechanisms. J Physiol (Lond). 2016;594(5):1215-1230

3. McIntire DD, Bloom SL, Casey BM, Leveno KJ. Birth weight in relation to morbidity and mortality among newborn infants. $N$ Engl J Med. 1999;340(16):1234-1238

4. Barker DJ, Gelow J, Thornburg K, Osmond C, Kajantie E, Eriksson JG. The early origins of chronic heart failure: impaired placental growth and initiation of insulin resistance in childhood. Eur J Heart Fail. 2010;12(8):819-825.

5. Visentin S, Grumolato F, Nardelli GB, Di Camillo B, Grisan E, Cosmi E. Early origins of adult disease: low birth weight and vascular remodeling. Atherosclerosis. 2014;237(2):391-399.

6. Barker DJ, Osmond C, Golding J, Kuh D, Wadsworth ME. Growth in utero, blood pressure in childhood and adult life, and mortality from cardiovascular disease. BMJ. 1989;298(6673):564-567.

7. Barker DJ, Osmond C, Simmonds SJ, Wield GA. The relation of small head circumference and thinness at birth to death from cardiovascular disease in adult life. BMJ. 1993;306(6875):422-426.

8. Barker DJ, Winter PD, Osmond C, Margetts B, Simmonds SJ. Weight in infancy and death from ischaemic heart disease. Lancet. 1989;2(8663):577-580.

9. Fall CH, Vijayakumar M, Barker DJ, Osmond C, Duggleby S. Weight in infancy and prevalence of coronary heart disease in adult life. BMJ. 1995;310(6971):17-19.

10. Partridge EA, et al. An extra-uterine system to physiologically support the extreme premature lamb. Nat Commun. $2017 ; 8: 15112$.

11. Hunter DS, Hazel SJ, Kind KL, Owens JA, Pitcher JB, Gatford KL. Programming the brain: Common outcomes and gaps in knowledge from animal studies of IUGR. Physiol Behav. 2016;164(Pt A):233-248.

12. Back SA, Riddle A, Dean J, Hohimer AR. The instrumented fetal sheep as a model of cerebral white matter injury in the premature infant. Neurotherapeutics. 2012;9(2):359-370.

13. Penning DH, Grafe MR, Hammond R, Matsuda Y, Patrick J, Richardson B. Neuropathology of the near-term and midgestation ovine fetal brain after sustained in utero hypoxemia. Am J Obstet Gynecol. 1994;170(5 Pt 1):1425-1432.

14. Tchirikov M, Strohner M, Scholz A. Cardiac output and blood flow volume redistribution during acute maternal hypoxia in fetal sheep. J Perinat Med. 2010;38(4):387-392.

15. Kamitomo M, Longo LD, Gilbert RD. Right and left ventricular function in fetal sheep exposed to long-term high-altitude hypoxemia. Am J Physiol. 1992;262(2 Pt 2):H399-H405.

16. Reller MD, Morton MJ, Giraud GD, Reid DL, Thornburg KL. The effect of acute hypoxaemia on ventricular function during beta-adrenergic and cholinergic blockade in the fetal sheep. J Dev Physiol. 1989;11(4):263-269. 
17. Hernandez-Andrade E, Benavides-Serralde JA, Cruz-Martinez R, Welsh A, Mancilla-Ramirez J. Evaluation of conventional Doppler fetal cardiac function parameters: E/A ratios, outflow tracts, and myocardial performance index. Fetal Diagn Ther. 2012;32(1-2):22-29.

18. Poudel R, McMillen IC, Dunn SL, Zhang S, Morrison JL. Impact of chronic hypoxemia on blood flow to the brain, heart, and adrenal gland in the late-gestation IUGR sheep fetus. Am J Physiol Regul Integr Comp Physiol. 2015;308(3):R151-R162.

19. Wheaton WW, Chandel NS. Hypoxia. 2. Hypoxia regulates cellular metabolism. Am J Physiol, Cell Physiol. 2011;300(3):C385C393.

20. Rossidis AC, et al. Chronically hypoxic fetal lambs supported by an extra-uterine device exhibit mitochondrial dysfunction and elevations of hypoxia inducible factor 1-alpha. Fetal Diagn Ther. 2018;1:1-8.

21. Vranas S, et al. Small size at birth predicts decreased cardiomyocyte number in the adult ovine heart. J Dev Orig Health Dis. 2017;8(5):618-625.

22. Jonker SS, Louey S, Giraud GD, Thornburg KL, Faber JJ. Timing of cardiomyocyte growth, maturation, and attrition in perinatal sheep. FASEB J. 2015;29(10):4346-4357.

23. Patterson AJ, Zhang L. Hypoxia and fetal heart development. Curr Mol Med. 2010;10(7):653-666.

24. Comas M, Crispi F, Cruz-Martinez R, Martinez JM, Figueras F, Gratacós E. Usefulness of myocardial tissue Doppler vs conventional echocardiography in the evaluation of cardiac dysfunction in early-onset intrauterine growth restriction. Am J Obstet Gynecol. 2010;203(1):45.e1-45.e7.

25. Crispi F, et al. Cardiac dysfunction and cell damage across clinical stages of severity in growth-restricted fetuses. Am J Obstet Gynecol. 2008;199(3):254.e1-254.e8.

26. Fall $\mathrm{CH}$, et al. Fetal and infant growth and cardiovascular risk factors in women. BMJ. 1995;310(6977):428-432.

27. Li G, Xiao Y, Estrella JL, Ducsay CA, Gilbert RD, Zhang L. Effect of fetal hypoxia on heart susceptibility to ischemia and reperfusion injury in the adult rat. J Soc Gynecol Investig. 2003;10(5):265-274.

28. Li G, Bae S, Zhang L. Effect of prenatal hypoxia on heat stress-mediated cardioprotection in adult rat heart. Am J Physiol Heart Circ Physiol. 2004;286(5):H1712-H1719.

29. Bartoli CR, et al. Bovine model of chronic ischemic cardiomyopathy: implications for ventricular assist device research. Artif Organs. 2013;37(12):E202-E214.

30. Hornick MA, et al. Toward physiologic extracorporeal support of the premature infant: technical feasibility of umbilical cord cannulation in mid-gestation fetal lambs. J Am Coll Surg. 2016;223(4S1):S91-S92. https://doi.org/10.1016/j.jamcollsurg.2016.06.182.

31. Hornick MA, et al. Umbilical cannulation optimizes circuit flows in premature lambs supported by the EXTra-uterine Environment for Neonatal Development (EXTEND). J Physiol (Lond). 2018;596(9):1575-1585.

32. Partridge EA, et al. An extra-uterine system to physiologically support the extreme premature lamb. Nat Commun. 2017;8:15112.

33. Battaglia FC, Meschia G. Principal substrates of fetal metabolism. Physiol Rev. 1978;58(2):499-527.

34. Zhu MY, et al. The hemodynamics of late-onset intrauterine growth restriction by MRI. Am J Obstet Gynecol. $2016 ; 214(3): 367$. e1-367.e17.

35. Sun L, et al. Reduced fetal cerebral oxygen consumption is associated with smaller brain size in fetuses with congenital heart disease. Circulation. 2015;131(15):1313-1323.

36. Itskovitz J, LaGamma EF, Rudolph AM. Effects of cord compression on fetal blood flow distribution and $\mathrm{O}_{2}$ delivery. Am $J$ Physiol. 1987;252(1 Pt 2):H100-H109.

37. Pacileo G, Paladini D, Pisacane C, Palmieri S, Russo MG, Calabrò R. Role of changing loading conditions on atrioventricular flow velocity patterns in normal human fetuses. Am J Cardiol. 1994;73(13):991-993.

38. Bartoli CR, Wead WB, Giridharan GA, Prabhu SD, Koenig SC, Dowling RD. Mechanism of myocardial ischemia with an anomalous left coronary artery from the right sinus of Valsalva. J Thorac Cardiovasc Surg. 2012;144(2):402-408.

39. Kang J, et al. Continuous-flow LVAD support causes a distinct form of intestinal angiodysplasia. Circ Res. 2017;121(8):963-969. 